\title{
Cuando el turismo invade el Centro Histórico: Santiago de Querétaro
}

\author{
Daniel Hiernaux-Nicolas* \\ Carmen Imelda González Gómez**
}

Resumen. La búsqueda del crecimiento económico de las ciudades a partir del desarrollo de actividades turísticas se ha vuelto una constante en numerosas urbes del mundo. En este trabajo se analiza el proceso de «turistificación» del Centro Histórico de la ciudad de Querétaro. Inicialmente se partió de la hipótesis de un proceso de transformación de ese centro por una gentrificación convencional, el estudio permitió identificar las políticas turísticas como las principales responsables de la turistificación intensiva analizada a detalle en el Barrio de la Cruz. Se muestra la dependencia de esas políticas dentro de los planteamientos internacionales sobre patrimonio y su relación con el turismo, la carencia de propuestas originales de la clase política local y los crecientes efectos perversos que la invasión del espacio central queretano genera sobre la población, en particular sobre la de menores ingresos que es progresivamente expulsada del centro, con lo que se cumple de esta manera con una gentrificación inducida.

Palabras clave: UNESCO, turistificación, gentrificación, políticas patrimonialistas, Querétaro.

*Profesor investigador, Facultad de Ciencias Políticas Sociales, Universidad Autónoma de Querétaro. Correo-e: danielhiernaux@gmail.com

" Profesora investigadora, Facultad de Ciencias Políticas Sociales, Universidad Autónoma de Querétaro.Correo-e: carmenimelda@gmail.com 


\section{When tourism invades the Historic Center: Santiago de Querétaro}

Abstract. The quest for economic growth in cities through the development of tourism has become a constant characteristic of many cities across the globe. This article analyzes the process of touristification of the Historic Center in the city of Queretaro. Drawing on a hypothesis of a transformation process of conventional gentrification in that city, it allows for the identification of tourism policies such as those responsible for intensive touristification examined in detail within the Barrio de la Cruz neighborhood. It reveals a dependence on those policies from an international perspective on heritage and its relation to tourism, the lack of innovative proposals from the local political class and the increasingly negative effects with the encroachment on Queretaro's central spaces by the population, particularly those of lower incomes, who are increasingly being pushed out of the city center, and which results in an induced gentrification.

Keywords: UNESCO, touristification, gentrification, heritage policies, Queretaro. 


\section{Introducción}

Cuando crece de manera sustancial la «turismofobia», como se presencia en la actualidad en diversos países de Europa, se vuelve urgente reflexionar sobre los efectos del turismo en los espacios y las comunidades en los cuales se inserta. Vale anotar una clara diferencia entre los grandes destinos de playa y los destinos interiores. En el primer caso, los turistas suelen desempeñarse en espacios turísticos de alojamiento y zonas ad hoc de ocio y recreación. Por lo mismo, su contacto con los residentes permanentes es escaso y limitado en tiempo e intensidad; en ciertos casos, los habitantes del lugar se han desplazado hacia espacios más alejados, en cierta forma periféricos — social y espacialmente - a los espacios turísticos.

Muy diferente es la situación de los centros urbanos densamente poblados: todas las grandes ciudades conocen serios conflictos por la difusión de los turistas en espacios antes reservados a los residentes permanentes. Pensemos por ejemplo en París, donde el área central está sometida a una ocupación permanente de turistas que afluyen todo el año hasta llegar a un récord estimado para el año 2020 a veinte millones de turistas. Con esas cuantías, aun repartidas durante todo el año, y lo reducido en área de los distritos turísticos en comparación con el resto de la ciudad, es evidente que se manifiesta un problema de sobrepoblación que se expresa de manera más aguda en espacios emblemáticos como la Torre Eiffel, el Museo del Louvre y algunas avenidas de referencia para el consumo turístico como los Campos Elíseos y la rue de Rivoli (Pearce, 1998).

Similar situación se vive en Barcelona, que después de considerarse un modelo a seguir en cuanto a proyecto urbano así como a perspectivas 
turísticas, se está volviendo el ejemplo de lo que no hay que permitir: ${ }^{1}$ la llegada de cruceros que expelen miles de pasajeros a la vez, dispuestos a pasear a lo largo de la Rambla; el creciente turismo de congreso; la multiplicación salvaje de los alojamientos turísticos por gracia de las relaciones entre locales y turistas propiciadas por la nueva economía turística en la nube (tipo Airbnb), entre otros factores, están provocando no sólo un profundo malestar de los residentes sino también una reacción fuerte de algunos grupos decididos a responder pacíficamente — por el momento- a lo que consideran como una auténtica invasión.

Con toda evidencia, en México no se llega todavía a semejantes extremos, pero no está de más analizar el fenómeno con el fin de elaborar políticas sensatas para frenar los abusos y mitigar las cargas extremas que el turismo genera sobre las poblaciones locales.

\section{Querétaro: ¿una ciudad en gentrificación?}

En esta ocasión presentaremos algunos hallazgos de un estudio del Centro Histórico de la ciudad de Querétaro, en el área central de México, misma que desde unos pocos años atrás está sometida a presiones derivadas de las políticas urbanas de las autoridades locales asociadas con el incremento del turismo.

El objetivo central de la investigación fue analizar el caso del Barrio de la Cruz, uno de los barrios más emblemáticos del Centro Histórico de la ciudad de Querétaro, el cual se encuentra sometido a presiones hacia

${ }^{1}$ Véase el documental de Eduardo Chibás Fernández (2014), Bye bye Barcelona, España, en https://www.youtube.com/watch?v=kdXcFChRpmI 
lo que se ha llamado una "gentrificación», es decir, la expulsión de la población actual por la llegada de nuevos habitantes proveídos de un capital cultural y económico superior a los residentes tradicionales (Smith, 1996).

Sin embargo, a lo largo de la investigación se hizo evidente que la posible gentrificación debía estudiarse en asociación con otros aspectos: la protección del patrimonio, el branding de la ciudad —la promoción de la ciudad y del Centro Histórico como si fuera una marca comercial— efectuado por las autoridades, y el hecho de que se esté integrando una suerte de distrito turístico que recubre no sólo el Barrio de la Cruz, sino una parte creciente del Centro Histórico de la capital queretana.

Querétaro es una de las ciudades más dinámicas del sistema urbano mexicano. Su ubicación geográfica en el centro del país hacia Estados Unidos implica que sea cruzada por muy importantes vías de comunicación con tráfico intenso, a la vez que se ha vuelto un significativo nodo geográfico para situar empresas ligadas tanto a la Ciudad de México - como proceso de desconcentración de la misma o como empresas de nuevo cuño- como a los mercados estadounidenses y norteamericanos en general.

El crecimiento demográfico de la ciudad ha sido intenso y a la fecha se estima su población en alrededor de 900 mil habitantes en la ciudad capital y sus inmediaciones directas y un millón 400 mil en la llamada área o zona metropolitana que engloba cuatro municipios intensamente articulados con la capital. De esta manera, la demanda actual y potencial de vivienda es muy alta, por lo que se ha generado una actividad inmobiliaria muy intensa con una oferta de viviendas para diversos niveles socioeconómicos en fraccionamientos dispersos en toda la zona metropolitana, generando así una ciudad fragmentada, difusa y cada vez más caótica (González, 2015). 
El Centro Histórico, que se había mantenido en un estado casi letárgico hasta la década de 1970, se ha visto integrado en una serie de procesos de transformaciones a veces antagónicas: por una parte, una fuerte protección de un patrimonio construido considerable y notorio; por la otra, una intensa transformación física por procesos inmobiliarios generados por la conversión de edificaciones tradicionales en hoteles-boutique, restaurantes, bares y demás comercios y servicios; finalmente debe agregarse a esos procesos una mutación social por la presencia de numerosos visitantes: habitantes metropolitanos, trabajadores internacionales en residencia temporal, excursionistas en buena medida del Área Metropolitana de la Ciudad de México y turistas nacionales e internacionales.

El trabajo de campo de nuestra investigación en el Barrio de la Cruz se realizó de 2013 a 2015 en diversas etapas correspondientes a varios financiamientos, obtenidos de fondos de la Universidad Autónoma de Querétaro (UAQ), y prosigue a manera de un observatorio urbano microlocal. A lo largo de esas diversas etapas de la investigación, se realizaron levantamientos exhaustivos en campo, trabajo cartográfico, muestra censal, entrevistas abiertas no estructuradas y videograbadas, además del ineludible trabajo de gabinete sobre informes oficiales, notas de prensa, etcétera.

Por distintas razones que sería largo enumerar en este ensayo, se partió de la intención de comprobar la presencia de un proceso conocido como «gentrificación», esto es, como se mencionó antes, un desplazamiento de la población provocado por la llegada de nuevos residentes con mejores condiciones económicas, un capital cultural más elevado y, posiblemente, por lo menos para parte de ellos, una propensión a ubicarse en actividades que Richard Florida denominó «creativas» (Florida, 2002). Sobre la gentrificación existe un sinnúmero de estudios anglosajones que documentan 
casos muy variados (consultar entre otros la extensa y reciente bibliografía de Lees et al., 2016). Muy particularmente se ha observado entre ellos una propensión evidente a tratar el tema del recambio de población a partir de los conceptos marxistas de clase y poder, y algunos otros neomarxistas como la «desposesión», siguiendo en eso al geógrafo británico David Harvey.

Si bien es innegable que existe un proceso de desplazamiento de población y un reemplazo de la misma en numerosos centros históricos por el mundo, en países como México se observan además fenómenos distintos como lo hemos analizado en otros trabajos (véase entre otros Hiernaux, 2016; Hiernaux y González, 2014a) y que parecerían más significativos que el «poder del dinero» de estratos más favorecidos, capaz de sacar a los residentes actuales de sus barrios. Una cautela mayor se hacía entonces indispensable y el enfoque del trabajo se volvió cada vez más inductivo, en vez de la vía deductiva que se presentaba como evidente en un principio.

En otros términos, el amplio trabajo de campo que realizamos permitió poner en evidencia diversos procesos relacionados, entre otros, con una fuerte intervención del Estado a través de las autoridades locales que se origina en el seguimiento de ciertas ideologías tanto patrimonialistas como desarrollistas que se aplican casi sin cambios en una multiplicidad de centros de ciudades mexicanas. Es en ese contexto que el turismo apareció como uno de los principales factores de transformación de los centros históricos, lo que analizaremos en los siguientes incisos.

Como recién lo señalamos, algunas pautas ideológicas han tenido un fuerte arraigo entre los funcionarios locales, reflejando así no sólo orientaciones promulgadas desde arriba que emanan del discurso del gobierno federal, sino de las declaraciones de intención y políticas formuladas por algunas instancias internacionales. 
La primera que conviene citar es la Organización de las Naciones Unidas para la Educación, la Ciencia y la Cultura (UNESCO, por sus siglas en inglés), la cual se ha erigido en paladín de la protección patrimonial desde las postrimeras de la segunda guerra mundial. El discurso de esta institución se sustenta en la afirmación de que existen bienes comunes a toda la humanidad, que es preciso conservarlos de manera solidaria entre países, con normas y enfoques que se presentan como universales. De esta manera, y a través de su brazo ejecutivo, el Consejo Internacional de Monumentos y Sitios (Icomos, por su acrónimo en inglés), la UNESCO se ha dado a la tarea de nombrar edificaciones y sitios urbanos completos en una lista del Patrimonio Cultural de la Humanidad, a cambio de imponer condiciones drásticas de calidad y preservación que implican, entre otros factores, la presentación y la aplicación de un plan de manejo que deba ser autorizado por la institución, so pena de recibir amonestaciones o peor de revertirse el nombramiento.

En otro trabajo hemos analizado el hecho de que en México se han perseguido metas de protección patrimonial desde fechas tempranas en el siglo XX, mismas que fueron asumidas, replicadas y hasta mejoradas por las autoridades estatales de Querétaro (González y Hiernaux, 2015). También, hemos señalado que las normas y las reglas de operación relacionadas con el patrimonio que surgen a partir del nombramiento dado por la UNESCO, ${ }^{2}$ no sólo son rígidas, sino contradictorias con las políticas de descentralización del gobierno mexicano que pretende otorgar mayores poderes de decisión a las autoridades locales. Esta suerte de internacionalización de las políticas patrimoniales es un factor importante que ha provocado además una

2 En el caso de Querétaro, la adscripción del Centro Histórico en la lista del Patrimonio Cultural de la Humanidad data de 1996. 
homogeneización creciente de las políticas, así como de los usos y las mejoras introducidas en los centros históricos y finalmente de su apariencia.

Es evidente entonces que se ha difundido en México un imaginario patrimonial con fuertes tintes internacionalistas. Si bien las declaraciones más recientes sobre la protección del patrimonio inmaterial —entre otros, en la gastronomía y las artes - parecerían suavizar estas orientaciones, no es menos cierto que las políticas patrimonialistas mexicanas se han vuelto en buena medida el reflejo de los dictados internacionales.

A ello se debe añadir que el discurso patrimonialista ha escondido una propuesta, posiblemente más perversa, que es plantear la rentabilización de los centros históricos por medio del turismo: la ecuación «más turismo = mayor protección patrimonial» ha sido promovida como regla de oro por la UNESCO desde los 1960 (Hiernaux y González, 2015). El discurso neoliberal se ha aprovechado de esta relación sin discutirla, planteando que los centros de las ciudades son espacios con serias ventajas comparativas y que es recomendable aprovecharlas, no sólo para el turismo, pero sí incluyéndolo como un eje de desarrollo de los negocios (Porter, 1995).

No hay duda, y lo hemos podido comprobar a través de las entrevistas realizadas a residentes y a funcionarios, las tablas de la ley para las autoridades locales de Querétaro comprenden dos verdades indiscutibles desde su perspectiva: la primera, que el centro tiene que ser protegido; la segunda, que debe dedicarse al turismo.

La radicalidad de esta propuesta ha sido sostenida por la anterior administración municipal priista y por la actual panista, y nada indica hasta la fecha que se pudiera revertir. El grado extremo ha sido una declaración de un funcionario local que indicaba a una residente inconforme que el centro era para turistas y no para habitarlo. 
Con este sustento ideológico, las obras realizadas por las autoridades se explican por sí solas: la remodelación del Jardín Guerrero, una plaza tradicional al oeste del centro y con una frecuentación más bien popular, fue transformada para volverla una suerte de espacio de paz, prosperidad y familia, como lo enseña una representación oficial, casi caricatural, de la plaza remodelada con algunos personajes y el castillo de Disney, publicada por el municipio. La reacción popular no se hizo esperar y obligó a cambiar el diseño de ese espacio público, entre otros se conservó una fuente tradicional que iba a ser reemplazada por fuentes brotantes del piso, modelo trillado observable por todas partes (Hiernaux, González y Duering, 2016).

Luego, la Plaza Fundadores, en el corazón del Barrio de la Cruz, sufrió semejante martirio. Su remodelación la dejó poco deseable, como lo manifiesta la población. Varias calles del centro están en proceso de repavimentación para integrar un circuito turístico peatonal que irá del oriente (los conocidos Arcos y el Convento de la Cruz) hasta al oeste (la iglesia de Santa Rosa de Viterbo y el Jardín Guerrero).

Finalmente - aunque no de modo definitivo porque faltan muchos atropellos más programados a expensas de la ciudad y de la ciudadaníala Alameda está sujeta a remodelación y se prevé la construcción de un restaurante cuyo planteamiento deja presagiar que no será destinado a las clases populares que suelen disfrutar de este espacio, sino a la población de niveles medio y alto que pronto poblará los departamentos integrados al nuevo centro comercial-área residencial «Latitud la Victoria», a la fecha en proceso acelerado de construcción y situado a menos de un kilómetro del citado jardín.

La adecuación del Centro Histórico no puede pensarse, en la estrecha mente de los funcionarios, sin la eliminación de la población de bajos 
ingresos que encuentra trabajo en sus calles entre los residentes pero esencialmente entre los visitantes. La política de «higienismo urbano» que se ha ejecutado — sin que se haya formulado por escrito por razones obvias - han sido claras violaciones a los derechos humanos de la población local. Las personas sin techo que encuentran un descanso de fortuna en un rincón de una plaza o en la esquina del portón de una mansión colonial son expulsadas con violencia, en ocasiones usando mangueras de agua fría en pleno invierno.

Las mujeres indígenas que buscan vender en el centro sus muñecas tradicionales o los productos de sus saberes artesanales en el bordado, la cestería o la cerámica, son empujadas fuera del espacio central — de «vocación» turística - por inspectores que en ocasiones les roban («confiscan» según la terminología oficial) su mercancía. Suelen residir por unas noches en el albergue del Desarrollo Integral para la Familia (DIF, ubicado en la calle de Guerrero, a un costado de la casa municipal), pero de manera semipermanente en colonias fuera del recinto patrimonial durante la semana y regresan a sus pueblos el fin de semana (Vázquez y Prieto, 2013).

Los vendedores ambulantes que se ubicaban frente a la Alameda en espacios negociados con las autoridades municipales fueron violentamente desalojados para que se pudiera disfrutar de la entrada monumental de la misma del lado de la avenida Zaragoza. Los comerciantes se encuentran en ese momento en instancias judiciales contra las autoridades locales para regresarse a ese sitio o, como mínimo, encontrar una nueva ubicación digna y rentable.

No cabe duda de que las acciones represivas de las autoridades se aparentan con toda evidencia con la histórica tendencia higienista del siglo XIX que se aplicó en Europa: trazar nuevas avenidas, sacar a los «malvivientes», 
reprimir a toda costa fueron métodos bien conocidos y usados para imponer la modernidad en las ciudades europeas (Harvey, 2008). Las acciones de represión mencionadas no sólo repiten la historia del modelo europeo, sino que a la vez reproducen de manera provinciana las intervenciones del alcalde neoliberal Rudolph Giuliani en Nueva York (Smith, 1996) con su política de «tolerancia cero»; la misma fue validada, aunque se aplicó parcialmente, en la Ciudad de México por Andrés Manuel López Obrador en su paso por la gubernatura de la capital mexicana (2000-2006) y sigue en aplicación por sus sucesores perredistas hasta la actualidad, que aplican una ideología de centro-izquierda con profundas raíces neoliberales.

Todas estas intervenciones, que se entienden mejor dentro del marco de una protección patrimonialista y de la promoción del desarrollo local del Centro Histórico de Querétaro — a la par, ciertamente, de otros centros del país—-, se acompañaron de otras intervenciones no menos significativas: en primer lugar, se han autorizado un número creciente de hoteles-boutique en la ciudad y de establecimientos diversos claramente orientados al turismo.

La cantidad de establecimientos y cuartos en hoteles-boutique situados en el Centro Histórico es irrelevante en comparación con la fuerte construcción de hoteles de cadena que se ubican en varios puntos y ejes cruciales de la ciudad: a lo largo de la avenida 5 de febrero; en el nuevo desarrollo cercano a la central de autobuses y al estadio Corregidora, conocido como «Centro Sur» (véase Duering, 2015); en torno a la plaza comercial Antea, por el momento la mayor de la localidad y una de las mayores del país y, desde fechas recientes, hacia el nuevo aeropuerto internacional incrustado en un «clúster» aeroespacial considerado como una de las joyas de la corona modernizadora-industrial de Querétaro. 
Sin embargo, la autorización para operar esos hoteles de lujo es más que una oferta en sí, es antes que todo y también una intervención altamente simbólica sobre el espacio urbano del centro: está mostrando que él mismo es capaz de modernizarse, atendiendo requisitos de alta calidad de un turismo nacional e internacional de buenos recursos y exigente. $Y$ a la vez, se preserva el patrimonio otorgándole una «nueva vida» con el uso turístico, como lo ha pregonado por décadas la UNESCO. En cierta forma, entonces, los hoteles-boutique y los diversos establecimientos relacionados con el turismo y el ocio, que han proliferado en el Centro Histórico de Querétaro, son la vitrina elegante de una política desarrollista de nuevo cuño que no se sustenta solamente en la industrialización —aunque la consiente también - sino en la terciarización acelerada de la economía local.

A diferencia de la situación que prevalece en ciudades europeas donde son señalados negativamente los efectos perversos de la renta de espacios turísticos de persona a persona mediante el uso de las redes sociales y la internet como Airbnb, las autoridades queretanas parecerían no preocuparse por el crecimiento de esa oferta paralela (237 ofertas en el Centro Histórico de Querétaro al 11 de agosto de 2017), ${ }^{3}$ la cual no sólo es competencia directa de los establecimientos legalizados de alojamiento turístico sino que refuerza la ilegalidad de todo el sistema turístico. Desconcierta sobremanera esa ceguera hacia actos ilegales de empresarios cuando cualquier desvío, como los generados por la pobreza, es irremediablemente castigado por las autoridades: economía informal, venta callejera por

${ }^{3}$ En el Barrio de la Cruz se contaban 44 cuartos en hoteles diversos en 2013, contra 213 en 2015. Asimismo, hace un par de meses se habían detectado sólo 150 ofertas de Airbnb que merecen compararse con las 213 actuales; se nota un crecimiento espectacular en sólo dos meses. 
indígenas o habitar el espacio público por carencia de un techo propio. También es notorio que las autoridades se han hecho sordas a los reclamos intensos de los habitantes de algunas calles del Centro Histórico, quienes están desesperados por el ruido y los excesos de los usuarios de bares y lugares de diversión que han proliferado, en particular en el Barrio de la Cruz. Cabe destacar que algunas voces han indicado que las autorizaciones de bares fueron otorgados a familiares o prestanombres de funcionarios de administraciones anteriores, lo que explicaría muchas cosas.

En síntesis, se puede afirmar que no sólo existe una injerencia no sistemática pero intensa de la UNESCO, vía Icomos, que encuentra un relevo de mando en el Instituto Nacional de Antropología e Historia (INAH) del gobierno federal en contra de decisiones locales, y que tiene como motivo la aplicación de criterios no incluidos en la declaración patrimonial, como es la protección de la imagen paisajística desde la distancia. ${ }^{4}$

Es preciso aclarar que la UNESCO ha tenido que enfrentar conflictos con algunas ciudades que no pueden regirse por una política unidireccional a favor del turismo y del patrimonio: puede mencionarse el ejemplo de Dresden, capital del estado de Sajonia en Alemania, ciudad que requería un puente sobre el río para reducir la congestión del tráfico y que acabó saliéndose de la lista de Patrimonio de la Humanidad. El interés de los residentes tuvo prioridad sobre la protección patrimonial (en este caso el reclamo de la UNESCO se refería a la transformación del paisaje tradicional por la presencia del puente) y sus posibles efectos en el turismo urbano.

${ }^{4}$ En todo caso existe la figura de «zona de amortiguamiento», la cual generalmente consiste en un área complementaria a la que integra la declaración y en la que hay restricciones sobre edificaciones, en particular referidas a su altura. La misma existe en Querétaro. 
En la actualidad se presentan además «enérgicas reclamaciones» de la UNESCO por un desarrollo de más de trescientas casas en San Miguel de Allende, en el estado de Guanajuato, las cuales, según la institución, afectan el paisaje de la ciudad. Situación similar se presenta en Guanajuato, capital del estado, también por la edificación de un desarrollo habitacional que afectaría el paisaje del centro visto a la distancia y la percepción del entorno desde el mismo centro. Mientras tanto, semejante discusión no tiene curso en Querétaro, donde un número creciente de edificios en altura se hacen omnipresentes desde diversos sitios desde los cuales se goza de una visión panorámica de la ciudad.

Falta todavía referirnos al incremento de las visitas a la ciudad de Querétaro que ha tomado un curso acelerado en los últimos años. Ésta se puede explicar por diversas razones. La primera es sin lugar a duda la mejor imagen nacional e internacional de la ciudad generada por las políticas de construcción de la marca «Querétaro». Las operaciones de branding han sido numerosas y juegan con varios factores: la mayor seguridad para bienes y personas en el conjunto de la ciudad, frente a la creciente inseguridad que padecen no pocas ciudades mexicanas. De igual modo, la calidad del patrimonio urbano, un hecho irrebatible. La imagen usa a su favor la creación de nuevos empleos en ramas diversificadas, lo que es una realidad indiscutible, así como la creciente oferta de centros de consumo (centros comerciales) y más y mejores servicios de salud y educativos, entre otras ventajas. En síntesis, las autoridades afirman que en Querétaro se vive mejor, por lo que el lema «Feliz tú que vives en Querétaro» ha sido bien aceptado, lo que ha intensificado la migración definitiva de numerosas personas hacia la ciudad, pero también la inscripción de Querétaro en el mapa de los destinos deseados por excursionistas y visitantes de todo tipo. 
El crecimiento de la afluencia turística, demostrable por los datos oficiales al respecto, es el resultado de un proceso de reconfiguración geográfica de los flujos origen-destino del turismo en la región centro de México. Es bien conocido que la Ciudad de México — la actualmente denominada CdMx según los creadores de su nueva «marca» y los municipios conurbados- con su más de 20 millones de habitantes, es la mayor emisora de turistas en el país tanto hacia destinos nacionales como internacionales. La inseguridad creciente en los alrededores de la capital y más recientemente en la misma, ha provocado que los destinos tradicionales cercanos hayan perdido su atractivo: Cuernavaca, «la ciudad de la eterna primavera», se ha tornado «la ciudad de la eterna balacera». La inseguridad es permanente también en destinos del Estado de México, como Valle de Bravo y en toda esa entidad, donde se multiplican — entre otros — los feminicidios. En este contexto, a dos horas y media de la capital, el estado de Querétaro se ha vuelto un destino atractivo, lo que se ve reforzado por el imaginario de seguridad, buena vida y calidad, que se ha construido en las dos últimas décadas.

De tal suerte, los «atractivos turísticos» situados en el Centro Histórico se maridan con otros como la Ruta del Queso y Vino, una construcción meramente virtual montada sobre unos pocos viñedos más orientados al turismo que a la vitivinicultura y la tradicional producción artesanal de quesos, con el destino balneario de Tequisquiapan, el Pueblo Mágico de Bernal y diversas haciendas coloniales que forman el hinterland turístico de la capital queretana.

El Centro Histórico de Querétaro se encuentra, como se demostró en las páginas anteriores, redefinido por la intencionalidad de promover el turismo y las políticas públicas en la materia que han derrochado mucho 
dinero para adecuar el espacio público a los requisitos explícitos e implícitos de su inscripción al Patrimonio Cultural de la Humanidad. Esto se ha hecho a pesar de los reclamos permanentes de los residentes, quienes están cada vez más exasperados por los atropellos y las políticas de los gobiernos locales.

Podemos entonces regresar al tema de la eventual gentrificación del espacio central de la ciudad. Es innegable que existe una transformación de la base social del Centro, pero es mucho menos importante de lo que se pudiera pensar, toda vez que una variable detiene en parte el proceso: la fuerte identidad de la población local residente permanente. Esta identidad se sostiene sobre la larga historia de sentirse parte de una comunidad donde no todos son nativos del lugar (52 por ciento de los residentes nacieron en el barrio, según nuestra encuesta), pero que sí la han adoptado como propia. Esta identidad se sustenta tanto en elementos tangibles como en el nacimiento in situ, la presencia de redes de familiares y amigos como lo pudimos observar por la encuesta que realizamos en 2015, la referencia al barrio para la compra de bienes y servicios —en específico la identificación con el mercado público - y la participación a una comunidad que mantiene ritos religiosos muy establecidos y de honda tradición.

Otro factor que juega un papel importante en el mantenimiento de la población local es el alto porcentaje de familias dueñas de su vivienda: 65 por ciento según nuestra estimación realizada sobre la base de la misma encuesta recién mencionada. Lo anterior provoca que se presentan menos casos donde los casatenientes puedan eliminar fácilmente a sus inquilinos al incrementar los alquileres, proceso que facilitaría la gentrificación como es el caso en países del norte. Más aún, si una familia decide vender su casa, realizará una ganancia alta por la fuerte alza de los precios inmobiliarios 
en los últimos años, por lo que el efecto de «desposesión» a la Harvey no tiene curso en el Barrio de la Cruz ni en el resto del Centro Histórico, salvo en contadas ocasiones.

Otro fenómeno digno de notarse es una presencia aún no muy significativa, pero creciente, de extranjeros en situación de segunda residencia o que ya optaron por hacer del Barrio de la Cruz su residencia principal. Lejos del modelo del turista desprendido de la localidad y de su población, los que residen en la Cruz han mostrado un sentido de lugar bastante comprometido, al grado de haber participado en protestas en contra de algunas políticas locales. Con la compra de su nueva residencia «mexicana», adquieren también una identidad diferente y comprometida con su entorno.

De igual manera merece señalarse que la UNESCO en la actualidad reflexiona sobre el riesgo mayor de la promoción del turismo en centros históricos: considera la posibilidad de que se esté generando una gentrificación de los mismos. Dicha vuelta de 180 grados de la institución se debe a la observación de auténticos desastres urbanos en no pocas ciudades, como es el caso de algunas europeas cuyos centros están invadidos por un turismo masivo, barato y poco respetuoso.

\section{Conclusiones}

La aceleración de la demanda de turismo urbano en México ha conducido a una creciente turistificación de los centros de las ciudades, espacios aptos para desarrollar actividades turísticas por la presencia de un fuerte patrimonio edificado y de tradiciones añejas. Este proceso conllevó a la 
necesidad de políticas públicas destinadas a adaptar el espacio público y a normar el espacio privado en función del destino deseado. Sin embargo, como se pudo ver, la falta de visión sobre las implicaciones y la poco inteligente decisión de seguir ciegamente los planteamientos de organismos internacionales y las experiencias foráneas, ha conducido a errores que apenas se empiezan a reconocer y que han provocado inclusive una turismofobia creciente.

Todavía más, las posturas dirigistas y carentes de capacidad de negociar asumidas por las autoridades frente a la población afectada, han provocado un divorcio creciente entre la sociedad civil y la clase política, hecho reforzado por la creencia — cierta o errónea- de que las obras emprendidas son fuentes de enriquecimiento ilícito de los funcionarios.

El caso queretano muestra que los habitantes del centro ya padecen molestias, desplazamientos y un forma de invasión de su espacio por el turismo, refiriéndonos no sólo a la presencia de los visitantes en la ciudad, sino a la vez por negocios que muestran un sello claramente orientado al turismo, desplazando las actividades tradicionales que respondían a las demandas cotidianas de la población.

Si bien la relación entre sociedad civil y clase política está agrietada, no existen manifestaciones de repudio tan fuertes como las que ocurren en otros ámbitos urbanos, pero el riesgo de verlas desarrollarse es latente y crecerá si no se toman las medidas adecuadas para limitar la «invasión» de espacios que son los «comunes» de una sociedad local que merece poder disfrutarlos sin ser empujada fuera de los mismos por presiones directas o indirectas.

Una situación de indecisión como la que se vive hoy es nefasta no solo para los residentes y el sistema democrático, y a la vez para el propio 
turismo por la «turismofobia» creciente que no debe tardar en insinuarse en el modelo o los modelos turísticos mexicanos. Contrariamente a lo que afirma Germán Porras Olalla (2017), que «el turismo necesita ser gestionado, no politizado», en el contexto actual es necesario un debate político de nivel donde participen todos los afectados y participantes del sistema turístico para decidir hasta dónde debe permitirse la expansión del modelo de desarrollo turístico en centros históricos, qué normas deben imponérsele (por ejemplo, en la regulación de los alojamientos informales), qué espacios pueden ocupar, entre otros temas. Si bien la politización por actos de repulsa no es la mejor vía, tiene la ventaja de mostrar dónde duele en la situación actual e invita a pasar a otra etapa, a la concertación política democrática y transparente.

\section{Referencias}

Duering, Emiliano (2015), Territorios difusos, murallas reales: una búsqueda de la segregación urbana, Centro Sur de la ciudad de Querétaro, Querétaro, Plaza y Valdés/Universidad Autónoma de Querétaro.

Florida, Richard (2002), The rise of the creative class: and how is transforming work, leisure, community and everyday life, Nueva York, Basic Books.

González, Carmen Imelda (coord.) (2015), Imaginarios y fragmentos de metrópoli: Querétaro, México, Querétaro, Universidad Autónoma de Querétaro.

González, Carmen Imelda y Daniel Hiernaux (2015), «Patrimonio y centralismo: perspectivas críticas desde el ámbito local», en Andrés Garrido del Toral (coord.), A 190 años del federalismo en México 1824-2014, Querétaro, Instituto de Estudios Constitucionales del Estado de Querétaro, pp. 313-367. 


\section{CuAndo el turismo inVAde el Centro Histórico}

Harvey, David (2008), París, capital de la modernidad, Barcelona, Akal.

Hiernaux, Daniel (2016), «La gentrificación «criolla` en México: entre el tipo ideal y las prácticas socio-espaciales en los centros históricos mexicanos», en Lulle, Thierry, Yasna Contreras y Oscar Figueroa (eds.), Cambios socioespaciales en ciudades latinoamericanas, ¿procesos de gentrificación?, Bogotá y Santiago, Universidad Externado de Colombia/Universidad de Chile, pp. 39-59.

Hiernaux, Daniel y Carmen Imelda González (2014b), «Gentrificación simbólica y poder en los centros históricos: Querétaro, México», Geocrítica, pp. 1-16. (2014a), «Turismo y gentrificación. Pistas teóricas sobre una articulación», Revista de Geografía Norte Grande (58), pp. 55-70. (2015), «Patrimonio y turismo en centros históricos de ciudades medias. ¿Imaginarios encontrados?», URBS. Revista de Estudios Urbanos y Ciencias Sociales, 5(2), pp. 111-125, en http://www2.ual.es/urbs/index.php/urbs/article/ view/hiernaux_gonzalez

Hiernaux, Daniel, Carmen Imelda González y Emiliano Duering (2016), «Nuevas políticas urbanas, gentrificación y resistencia: movimientos sociales incipientes en Querétaro», en Fernando Carrión y Jaime Erazo (coords.), El derecho a la ciudad en América Latina. Visiones desde la política, México y Ottawa, Universidad Nacional Autónoma de México/International Development Research Centre, pp. 241-257.

Lees, Loretta, Hyun Bang Shin y Ernesto López Morales (2016), Planetary gentrification, Cambridge, Polity Press.

Pearce, Douglas (febrero de 1998), «Tourist districts in Paris: structure and functions», en Tourism Management, 19(1), pp. 49-65.

Porras Olalla, Germán (2017), «El turismo necesita ser gestionado, no politizado», Hosteltur, en https://www.hosteltur.com/comunidad/005689_el-turismonecesita-ser-gestionado-no-politizado.html 
Porter, Michael (mayo-junio de 1995), «The competitive advantage of the inner ci-ty», Harvard Business Review, pp. 55-71.

Smith, Neil (1996), La nueva frontera urbana. Ciudad revanchista y gentrificación, Madrid, Traficantes de Sueños.

Vázquez, Alejandro y Diego Prieto (coords.) (2013), Indios en la ciudad. Identidad, vida cotidiana e inclusión de la población indígena en la metrópoli queretana, Querétaro y Ciudad de México, Universidad Autónoma de Querétaro/Instituto Nacional de Antropología e Historia. 